\title{
Téoros
}

Revue de recherche en tourisme

\section{La gestion de l'entreprise touristique au-delà de la gestion traditionnelle}

\section{Alain Flautre}

Volume 5, numéro 3, novembre 1986

La gestion touristique : les nouvelles technologies

URI : https://id.erudit.org/iderudit/1080531ar

DOI : https://doi.org/10.7202/1080531ar

Aller au sommaire du numéro

Éditeur(s)

Université du Québec à Montréal

ISSN

0712-8657 (imprimé)

1923-2705 (numérique)

Découvrir la revue

Citer cet article

Flautre, A. (1986). La gestion de l'entreprise touristique au-delà de la gestion traditionnelle. Téoros, 5(3), 2-5. https://doi.org/10.7202/1080531ar d'utilisation que vous pouvez consulter en ligne.

https://apropos.erudit.org/fr/usagers/politique-dutilisation/ 


\title{
La gestion de l'entreprise touristique au-delà de la gestion traditionnelle
}

\author{
par Alain Flautre*
}

Les années 1970 ont vu l'avènement d'un. véritable phénomène de civilisation: le "management". .

Or ce phénomène est profondément imprégné par des préoccupations propres à la grande entreprise voire le plus souvent a la tres grande organisation diversifice ou non et a l'organisation multinationale. Les règles de base sont alors la croissance, croissance en part de marché, en volume de ventes, en terme de profit.

Les outils spécifiques requis par les tâchesclé (production-marketing-comptabilitéfinance) sont connus et généralement mầtrises. En fait, selon cette optique l'organisation est la résultante de lạ coordination rationnelle des activités des individus qui la composent et qui permettent à celle-ci $\mathrm{d}^{+}$atteindre les buts et les objectifs fixés tout en optimisant la diversion du travail et des fonctions et en hiérarchisant l'autorité et les responsabilites.

Cette définition insiste, à juste titre, sur l'aspect formel des organisations, sur la distribution du pouvoir et sur l'orientation de l'activité par des objectifs; elle suppose une taille assez grande et aussi, le plus souvent, un marché oligopolistique. Mais la réalité touristique est tout autre, mis à part quelques organisations de grande taille (ex.: transporteurs, organismes gouvernementaux, etc.), l'industrie touristique, dans tous les secteurs qui la composent, est tres fragmentée; il y a peu de possibilités d'économie d'echelles et peu ou pas de barrière à l'entrée: ce sont les principales raisons de cette fragmentation. Comme ses consoeurs oeuvrant dans des industries de production, l'entreprise touristique fait face à un environnement turbulent et complexe où les systèmes sociaux exercent des pressions soit par des lois, des comportements d'achats, une évolution de la technologic, les contraintes financières, la concurrence; les partenaires ou tout simplement par les caractéristiques climatiques, historiques ou géographiques dans lesquelles cette organisation touristiques a decide de crottre.

*Alain Flautre, M.B.A. Arim. A. charué de cours en administration touristiqus, Conseiller affilies en admintstration et marketing touristique aupres des societs Woods-Gordon et Cégi da Montráal.

\section{La réalité touristique}

Derrière cette dimension réaliste de l'entreprise touristique se cache de façon plus prosaïque, la préoccupation de survie face à un environnement marqué par l'incertitude, le risque, les contraintes, les retournements brutaux, les remises en cause permanente. En fait, l'entreprise touristique doit, pour survivie, mettre en évidence ses caractéristiques différencielles face à la concurrence et à ses marchés.

L'entreprise touristique doit, comme d'autres entreprises de services, faire face au caractère intangible de l'industrie, it la multiplicité des services offerts et à la participation directe ou indirecte de la clientèle dans le processus de production.

Dû à cette participation, l'enviromement intrinsěque et principalement social et humain revêt une importance cruciale et l'implantation d'une stratégie de marketing interne fait partie intếgrante de la gestion touristique.
Il semble bien évident qu'il n'existe pas de modèle de gestion d'entreprise touristique tout au plus des "outils" qui perrinettent aux gestionnaires de mettre en oeuvre leur crếativité, leur innovation, leur dextérité à communiquer et à contrôler.

En fait, une organisation est la rencontre du style de son fondateur, de la mission donnée, des caractéristiques socioculturelles de ses environnements, des contraintes qu'elle subit, des opportunités qu'elle a su saisir, du système de valeur de l'equipe dirigeante et des objectifs économiques qu'elle s'est donnée.

La plupart des naufrages d'entreprises par "fuites en avant" incontrồlés ne résultentils pas de l'ignorance de ces relations simplistes? L'adaptation "anticipative" du contenu et de l'évolution des différentes activités face aux contraintes financières, humaines, politiques, juridiques, technologiques et climatiques revêt ainsi une importance cruciale au fur et à mesure que

\section{Tableau I}

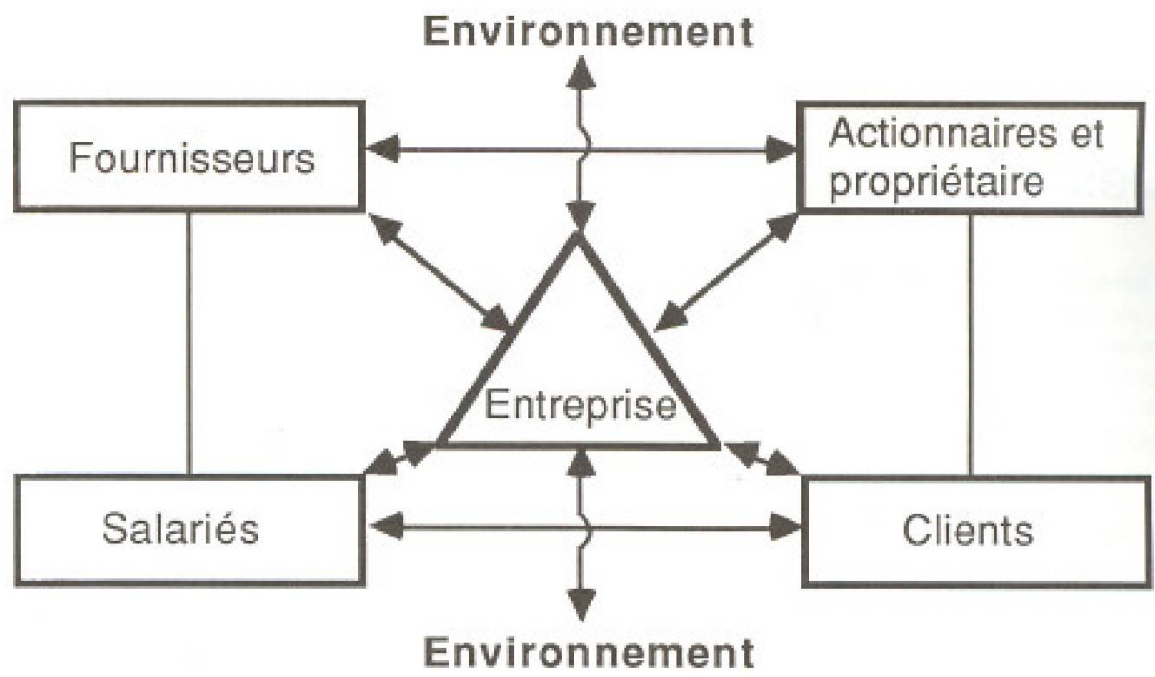

Eléments participants de la dynamique d'entreprise (1)

(1) Source: Le management des entreprises de services. J.P. Flipo 1984. 
s'impose l'inćluctable nécessité d'une gestion en avenir incertain et à haut niveau de risque.

\section{La gestion touristique}

En plus des points ci-haut mentionnés, l'intangibilité des services offerts, les contextes ct l'aspect social sont partie prenante de la gestion de l'entreprise touristique et demandent des techniques de gestion qui cortespondent à ces spécificités.

Une bonne stratégie d'entreprise devrait être concentrée autour de pôles fondamentaux:

- la stratégie corporative;

- le systeme de contraintes;

- les objectifs économiques;

- les stratégies de marketing.

La dimension stratégique constitue ainsi le rôle primordial de l'équipe dirigeante qui, à partir de l'évaluation continue de l'environnement extrinseque et intrinseque et de l'anticipation des changements internes ou externes, doit réaliser les conditions de cohérence des quatre pôles de la stratégie globale, tel que le montre le schéma numéro 1.

En effet toute incompatibilité entre trois des quatre póles crée indéniablement des déséquilibres stratégiques structurels qu'aucune mesure technique ne peut corriger.

D'où l'importance de conduire le développernent selon une approche itérative consistant à formuler la stratégie formelle qui permet de réduire les risques et la vulnérabilité de l'entreprise.

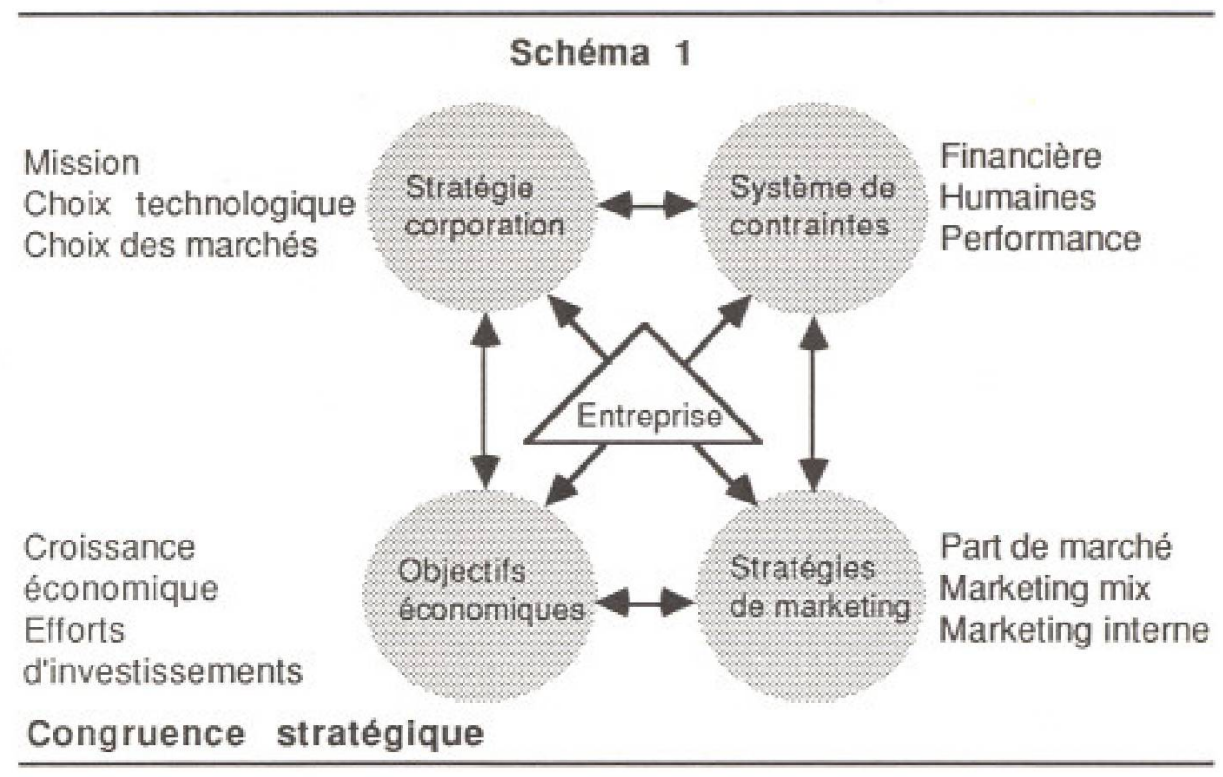

Ainsi le schéma conceptuel suivant (voir le schéma numéro 2) montre les actions auxquelles les gestionnaires doivent exécuter pour préparer la stratégie formelle de l'entreprise.

La strategie formelle reflète donc l'évaluation de l'equipe dirigeante face aux fateurs contextuels, structurels et internes, Il s'agit que cette équipe soit en mesure de se doter d'un système d'information adéquat pour lui donner les données les plus pertinentes face à ces facteurs. Il s'agit également de la part des dirigeants de mettre à l'epreuve leur sens de l'innovation pour concevoir des options inédites pour mettre en évia ses marches. dence les avantages différentiels de l'entreprise face à ses environnements et surtout

Avant de réaliser la stratếgie, l'équipe dirigeante, pour accroitre l'efficacite de l'actualisation de celle-ci, doit la communiquer à tout le personnel afin qu'elle soit comprise par tous ceux qui vont appliquer les politiques générales visant à réduire les écarts entre la stratégie formelle et la stratégie actualisée. Il faut comprendre que cette dimension a une importance primordiale dans une entreprise de services, comme l'entreprise touristique, car le personnel a un contact direct avec la clientêle. Le climat organisationnel sera done largement favorable à l'implantation de la stratégie si les individus ont participé à son ćlaboration (les gens réalisent génétalement ce qu'ils ont crét).

Mais là ne s'arrête pas la gestion de l'entreprise touristique. Il est nécessaire aux dirigeants de connaître la dynamique de secteur de l'industrie touristique dans laquelle ils se trouvent car chacun d'entre eux a ses propres caractéristiques même si tous font partie integrante de l'industrie touristique. Ainsi le secteur des agences de voyages a ses caractéristiques, comme les hôtels, les bases de loisirs, les transporteurs, les fourvoieries... ont les leurs.

La phase du secteur va lui aussi avoir une influence sur la gestion et sur le développement de l'entreprise. Certains secteurs peuvent parcourir toutes les ćtapes du cycle de vie (émergence, croissance, maturité, saturation, déclin) à un rythme rapide; d'autres pourtont se maintenir au stade de maturité (hotels de luxe) sans jamais passer à la phase de déclin. Gräce à l'adaptation face ầ ses marchés et à l'innovation.

L'innovation vạ "réoxygéner" I'entreprise et permettre à celle-ci de changer la dynamique même de l'industrie. Ainsi les res- 


\section{Schéma 2}

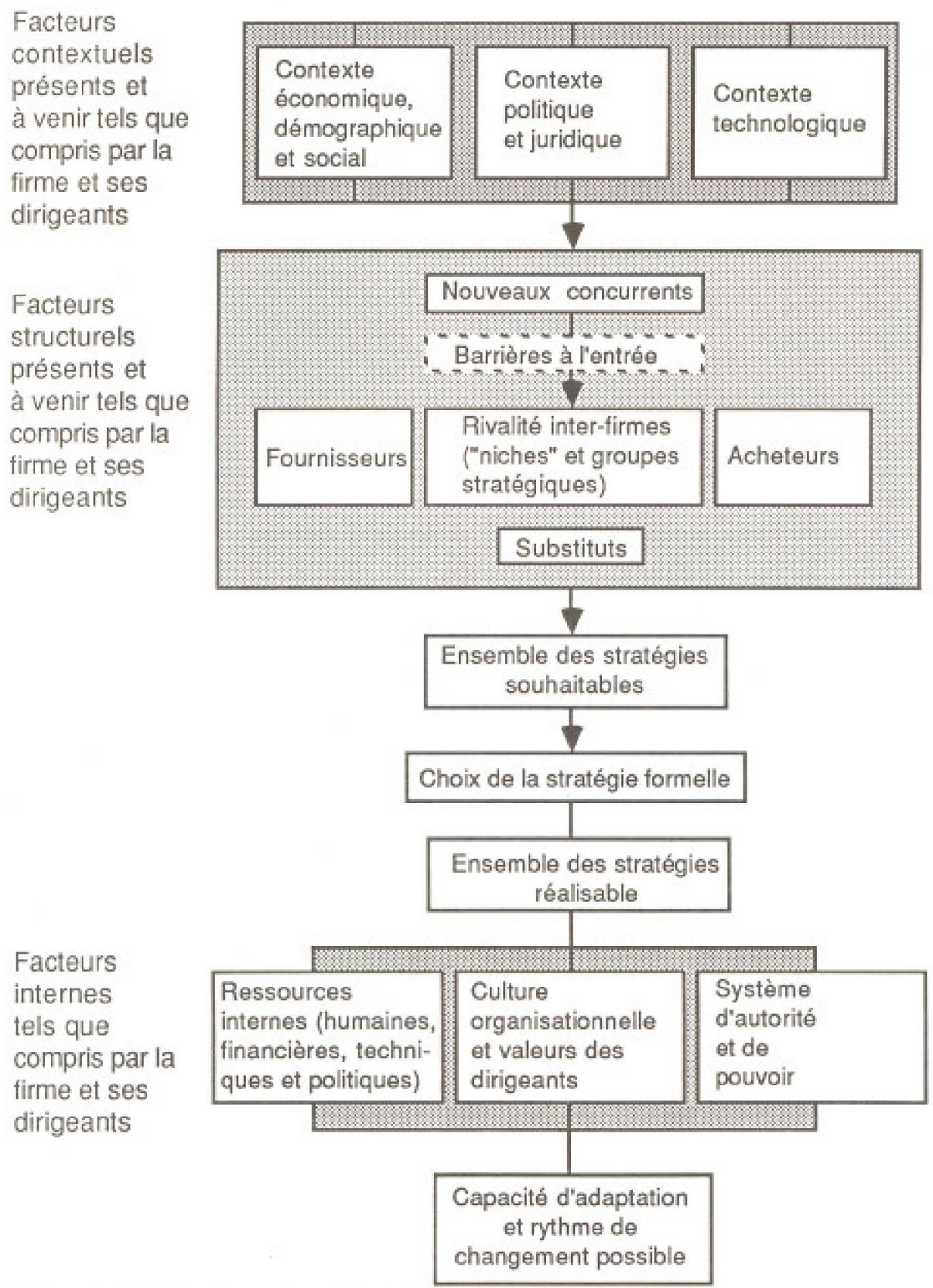

Allaire et Firsirotu: La gestion stratégique des organisations, 1984

taurants McDonald au debut des annees 60 , comprirent qu'une industrie intrinsequement fragmentée (faute d'économie d'échelles en particulier) représentait peu d'intérềt pour les investisseurs recherchant une forte croissance sur leur investissement. La standardisation et les stratégies de communication ont largement changer l'industrie de la restauration rapide et l'implantation d'un réseau de distribution via les franchises fit le succès légendaire de McDonald et la fortune de son innovateur M. Ray Krock.

L'industrie des agences de voyages qui est également très fragmentée et qui subit de fortes pressions de ses fournisseurs concurrents (transporteurs et voyagistes) ne peut esperer survivre dans sa forme actuelle, que grâce à l'innovation (regroupement, franchisage, innovation technologique) et en différenciant l'option stratégique par une publicité massive pour accroitté leur pénétration du marché des touristes et augmenter leur pouvoir de négociation face à leurs fournisseurs.

\section{Tangébilisation}

Les touristes, comme tous consommateurs, recherchent la sécurité d'autant plus qu'ils achètent dans un environnement. la plupart du temps inconnu. Son idé sur le service et la qualité de celui-ci se fait en trois étapes tel que le montre le schéma numéto 3 qui suit:

1. phase préc-achat:

2. réalisation du service:

3. phase post-achat.

Ainsi pour le choix d'un hôtel, façade, environnement immédiat, I'agencement du hall d'entrée et la personnalité de l'accueil sont les étapes de prés-achat. La nuit qu'il décire de passer dans le dit hôtel lui permet d'évaluer réellement la qualité de I'hótel, le lit, la décoration, la propreté, les services intégrés ou non dans sa chambre. la salle de bain, le service aux chambres, la téléphoniste, le mini bar, les services de nettoyeur... Bref tous ces éléments qui sont importants pour la satisfaction du client.

En tangibilisant les moindres indices, l'on donne aux clients des indications sur la quatlité des services offerts. C"est ainsi que les restaurants se positionnent sur le marche autant par leur decor, leur environnement que par la qualité et l'agencement des mets qui y sont servis.

L'élément humain est également un sujer de tangibilisation. A cet effet, la gestion des ressources humaines dans l'entreprise touristique joue un rôle crucial. Il est essentiel de susciter un climat organisationnel favorable a l'atteinte des objectifs fixés dont le plus important est la satisfaction du client.

\section{Le marketing}

L'implication marketing au sein de l'entreprise touristique revêt une dimension qui dépasse celle du marketing traditionnel. En effet le marketing touristique tient compte du contact direct client prestataire et de lat participation de ce client au processus de production. Nous sommes loin de la gestion marketing étudiée dans la plupart des manuels.

En fait, le marketing touristique s'il inclue les differentes variables du mix (service, publicité, prix, réseau de distribution) inclut également le marketing interne qui résulte du développement du pouvoir du personnel et particulièrement du personnel en contact avec la clientele. Les agents de réservation dans une agence de voyages peuvent développer le chiffre d'affaire de l'entreprise mais ils peuvent également le detruire. Au même titre qu'un serveur dans un restaurant ou un moniteur de ski dans une station. Il ne s'agit plus de pouvoir faire face à des thèmes syndicaux connus: salaires, avantages sociaux, conditions de travail mais à rendre un climat favorable pour la clientele pour développer l'entreprise tout en assurant la survie de celle-ci et de la leur. En fait, pour que le marketing touristique soit efficient il est essentiel de prendre compte du comportement du client, de 
l'influencer si celui-ci risque d'altérer la qualite du service rendu at de voir le personnel comme un client qui "achète" un emploi.

\section{La clientèle}

En dehors des etudes de marché visant à cerner la clientèle cible, ses comportements. et habitudes d'achat, des stratégies de communications appropriées, le support physique fait également partie de l'approche marketing surtout lorsque le service est délivré dans les locaux ou grâce au matériel du prestataire: hôtelier, restaurant, terrain de golf, station de ski, parc d'amusement. Le décor, l'agencement va créer l'atmosphère favorable aux décisions d'achat.

Les autres clients peuvent être perçus par le client individuel comme un coneurrent qui lui enlèvent une partie de l'attention du personnel d'autant plus si les "autres" clients sont perturbateurs. Qui n'a pas vécu l'expérience dans un restaurant où de joyeux lurons viennent perturber votre soirée intime... vous laissant une piètre impression des services de cet établissement.

\section{Le personnel contact}

Tout en produisant le service, le personnel doit le vendre. L'agent de réservation d'une compagnie aćrienne ne doit pas être simplement un "preneur de commande" il doit suggérer au client l'hôtel, la location de voiture, le circuit touristique, le transport entre l'aúroport et la résidence ou le lieu de destination, le tout en fonction du type de client de ses attentes de ses besoins. Celà implique de la part du personnel en contact des qualités relationnelles et une comprehension de la psychologie du client.

\section{La communication}

L'équipe dirigeante doit savoir communiquer, soit: tirer profit de la participation du client au processus de production afin de le satisfaire et de le "fidéliser". Ainsi les gestionnaires doivent faciliter cette communication sans toutefois tomber dans l'exoes qui pourrait entraîner des coûts excessifs. Ainsi le bureau de tourisme d'une région devrait, au lieu de multiplier le personnel de l'accueil, utiliser des médias électroniques pour inciter le touriste "en devenir" à séjourner dans tel ou tel autre endroit de la dite région. Ce type de communication "economique" est d'ailleurs de plus en plus utilisé (agences de voyages, palais des congres, club med). Le personnel devient alors un conseiller, un relationniste.

Les gestionnaires marketing doivent également tenir compte des capacités de communication du personnel contact, capacité qui devrait être prise en cause lors de l'engagement. Développer des séances de formation en communication pour ses employes. Utiliser cette communication interpersonnelle dans ses stratégies de publicité.

Schéma 3

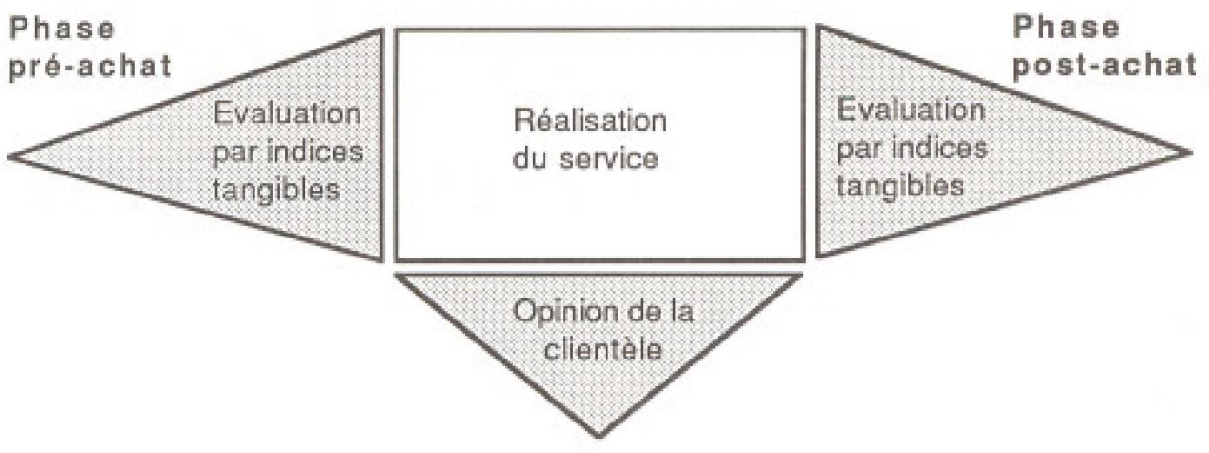

Source: Le management des entreprises de services. J.P. Flipo 1984.

Enfin I'entreprise devrait, dans la mesure du possible, laisser une marge de manoeuvre contrólec à son personnel en contact pour differencier les services offerts. Selon, Martin L. Bell c'est la prolongation naturelle de la strategie car: "Le tacticien accomplit l'acte de base du service, eduque le client dans le sens du comportement qui facilitera sa satisfaction finale, reçoit et réagit à la manière dont le service est uti= lisé, et modifie l'offre dans la limite où il est autorise ou habileté à le faire."

Ce sont là des "toutils" pour le gestionnaire qui veut s'assurer une part de marché dans son secteur de l'industrie touristique.

En dehors de ces facteurs, le gestionnaire d'entreprise touristique doit effectuer' les tâches usuelle d'administration: planifier, diriger, contrôler, motiver. Mais dans une économie qui connaît désormais des contraintes d'une ampleur et d'une complexité sans précédent depuis trois décennies, les gestionnaires voient dans la compression des dépenses une stratégie défensive dont le caractère prioritaire conditionnerait la performance de l'entreprise.

Le ralentissement de la croissance, la hausse des facteurs essentiels de production (énergie, salaires, services), les marges de manoeuvre réduites sur les hausses de prix de vente, les taux du loyer de l'argent, la nécessité d'efforts continus d'investissements, de recherche et de productivite, l'obsolescence rapide des equipements et de la technologie, la fluctuation des monnaies et enfin l'inflation créent un environnement où le levier "Économies" est à la fois impératif et critique.

Face à ce constat, les dirigeants prennent souvent des mesures réactives et parcellaires alors que la congruence stratégique nécessite une intégration permanente d'une fonction de prévention et de suivi des "glissements" des composantes de la structure. Cette philosophie simpliste en apparence est difficile à faire passer concrètement dans les faits. Seules les entreprises ayant des structures adaptatives et légères peuvent optimiser sur la mise en oeuvre des stratégies.

Dans cette approche, il appartient aux dirigeants d'entreprises touristiques d'introduire, d'animer, d'adapter, de contrôler et de faire développer au sein de l'organisation, par les responsables-clé, les stratégies.

Les trois I de la stratégie ne sont-ils pas:

- innovation;

- information:

- implantation

f

ALLAIRE et FIRSIROTU: La gestion strategique eds organisations: 1984.

La direction des entreprises - concepts et applications, MaGraw Hill, 1985.

Club I.C.G.: Vers une gestion participative, Hachette, 1977 .

DAVIDSON, David. S.: How to succeed in a service industry - Turn the organisation chart upside down. Management Review, avril 1979.

EIGLIER, P. et LANGEARD, E.; Le marketing des entreprises de services, Fevue française de gestion. mars-avril 1977.

FLIPO, Jean-Paul: Le management des entreprises de services, Les éditions d'organisation, 1994.

GEORGE, W.R, or KELLY J.P: Promotional activities for services are different, Business, août 1982. MILLER, Foger (dir): La direction des entreprises . concepts et applications, McGraw Hill, 1985.

PARIS, Francis: Missions stratégiques de l'équipe dirigeante, Bordas, 1980.

RISNY, M: Strucutre de l'entreprise et capacité d'innovation, Entreprise moderne d'édition, 1978.

SCHEINER, Benjamin: The service organization - $\mathrm{Cl}$ mate is crucial Organizational Dynamics, automne 1980. 